DOI: 10.1136/annrheumdis-2018-eular.6701

\section{SAT0359 EVOLUTION OF RENAL FUNCTION IN GOUT AFTER NORMALISATION OF SERUM URIC ACIDLEVEL}

\section{E. Gazaix-Fontaine ${ }^{1}$, A. Ostertag ${ }^{2}$, H.-K. Ea ${ }^{1}$, F. Lioté ${ }^{1} .{ }^{1}$ Rheumatology} Department, ${ }^{2}$ INSERM UMR 1132 Bioscar, Centre Viggo Petersen, Hospital Lariboisière, Paris, France

Background: Chronic kidney disease (CKD) is one of the main causes of mortality in gout. In addition, hyperuricemia can contribute to the decrease of renal function.

Objectives: The aim of this study was to evaluate the evolution of renal function after reducing serum uric acid (sUA) level to therapeutic target in gout, and to identify the factors that might influence this evolution.

Methods: Patients with gout were followed at a single university-based hospital. They were free of urate lowering therapy (ULT) and had an estimated glomerular filtration rate (eGFR) higher than $15 \mathrm{ml} / \mathrm{min}$. Renal function was measured at start of ULT and when sUA was reduced below $360 \mu \mathrm{mol} / \mathrm{L}$ (or $6.0 \mathrm{mg} / \mathrm{dL}$ ). ULT was allopurinol or febuxostat at the choice of clinician.

Results: In this retrospective study, 63 patients (pts) were included with an initial mean sUA level of $541 \mu \mathrm{mol} / \mathrm{l}( \pm 86)$. Overall 42 pts were treated with allopurinol and 21 with febuxostat for an average length of 278 days $( \pm 185)$. After ULT, mean eGFR differed significantly:+2.6 [IC95\%: $-0.279 ; 5.484 ; \mathrm{p}=0.08],+3$ [IC95\% $0.167 ; 5.794 ; p=0.04]$ and $+2,7$ [IC95\%: 0.490; 4.960; $p=0.02] \mathrm{ml} / \mathrm{min} / 1,73 \mathrm{~m}^{2}$ depending on the calculation method, Cockcroft-Gault (C-G), MDRD and CKDEPI, respectively. eGFR improvement was statistically significant, $p=0.04,0.02$ et 0.01 , respectively. Initial eGFR $>45 \mathrm{ml} / \mathrm{min} / 1,73 \mathrm{~m}^{2}$ was associated with a better outcome of eGFR expressed in percentage: $+4.7 \%$ vs $-7.3 \%(p=0.02),+6.3 \%$ vs $-4.9 \%(p=N S),+6.2 \%$ vs $-5.3 \%(p=0.03)$ according to the calculation method, $C-$ G, MDRD and CKD-EPI, respectively. We also found a better evolution of renal function when patients were treated with allopurinol compared to febuxostat, but confounding bias might have occurred since their mean eGFR was also better. In contrast, neither sex, BMI, presence of hypertension nor diabetes showed any significant effect on eGFR improvement.

Conclusions: Renal function was significant different after normalisation of sUA in gout patients. We observed an improvement of eGFR which is consistent with recent studies. Interestingly, when initial renal function was at CKD IIla level or higher (eGFR $>45 \mathrm{ml} / \mathrm{min} / 1,73 \mathrm{~m}^{2}$ ), renal outcome was even better. It strengthens the rationale to treat high sUA as soon as possible.

Acknowledgements: Thanks to Frédéric Lioté ${ }^{1,2,3}$, Hang-Korng $\mathrm{Ea}^{1,2,3}$ and Agnès Ostertag ${ }^{2}$

${ }^{1}$ Rheumatology Department and ${ }^{2}$ INSERM UMR 1132 Bioscar, Centre Viggo Petersen, (AP-HP) hospital Lariboisière; ${ }^{3}$ Univ. Paris Diderot, USPC, Paris, France

Disclosure of Interest: None declared

DOI: 10.1136/annrheumdis-2018-eular.3773

\section{SAT0360 A SUMMARY OF CLINICAL EVIDENCE FOR COMMONLY USED URICOSURICS FOR GOUT IN EUROPE}

F. Perez-Ruiz ${ }^{1}$, P. Kandaswamy ${ }^{2}$, R. Karra Gurunath ${ }^{2}$, H. Burton ${ }^{3}$, T. Jansen ${ }^{4}$. ${ }^{1}$ Rheumatology Division, Hospital Universitario Cruces, Bilbao, Spain; ${ }^{2}$ Grunenthal $\mathrm{GmbH}$, Aachen, Germany; ${ }^{3} \mathrm{DRG}$ Abacus, Bicester, UK; ${ }^{4}$ Rheumatology, VieCuri MC, Venlo, Netherlands

Background: Uricosurics (benzbromarone, probenecid, or sulfinpyrazone) are recommended for second-line treatment of gout if treatment target cannot be achieved with allopurinol or if allopurinol cannot be tolerated. While some uricosurics have been available since the 1970s, only two head-to-head trials have been conducted, both of which compared benzbromarone with probenecid. A new uricosuric, lesinurad, has recently been developed, but also has no head-to-head data against current uricosurics. There is therefore a need to compare the efficacy of uricosurics relative to each other via network meta-analysis (NMA), which requires high-quality evidence from well conducted trials, and also for evidencebased recommendations.

Objectives: To identify and assess the quality of the randomised controlled trial (RCT) evidence for uricosurics currently licensed for the treatment of gout, and to assess the feasibility of developing an NMA based on these trials.

Methods: A systematic review was conducted to identify RCT evidence for uricosurics, followed by an assessment of the quality of the comparisons possible based on this evidence using the GRADE system. This assessment was then used in a feasibility assessment for NMA based on the comparability of the trials in terms of population, comparators, and outcomes.
Results: In total, 21 publications reporting on 13 unique studies were identified by the SR, two of which had no common comparator arm with other trials and were excluded from further assessment. Eleven studies were included in the quality assessment informing comparisons between treatments for two outcomes: proportion of patients achieving SUA $<6 \mathrm{mg} / \mathrm{dL}$ (or $0.36 \mathrm{mMol} / \mathrm{L}$ ) and change from baseline in sUA. Issues with two or more elements of the methodological quality of the studies were present in all comparisons other than lesinurad plus allopurinol vs placebo plus allopurinol, including open-label design, incomplete reporting and sparse data mainly of safety assessment, and inadequate randomisation. The outcomes assessed in these RCTs were generally comparable across trials, but follow-up time ranged from 1 week to a mean of 19.6 months and few trials reported data at the same endpoint. In addition, line of therapy was unclear/not reported in four trials, with uricosurics used in a first-line population in two trials. The final GRADE quality rating was 5 (high) for lesinurad plus allopurinol vs placebo plus allopurinol and -1 to 1 (very low) for all other comparisons (febuxostat plus lesinurad was not included in comparisons). Based on this assessment, it was concluded that a NMA or other statistical comparison is not possible because of substantial heterogeneity between trials for methodological quality, outcomes, and population.

Conclusions: The quality of the randomised controlled trial evidence supporting the use of uricosurics other than lesinurad for gout is poor, and there is substantial heterogeneity in reported follow-up times and study populations. It is therefore not possible to make valid comparisons between these treatments based on this data, which may have implications for decision-makers in assessments of future treatments for gout.

Disclosure of Interest: None declared

DOI: 10.1136/annrheumdis-2018-eular.3026

\section{SAT0361 TRABECULAR BONE SCORE IN OSTEOGENESIS IMPERFECTA. IS IT USEFUL?}

H. Florez ${ }^{1}$, A. Muxi ${ }^{2}$, E. Gonzalez ${ }^{3}$, A. Monegal' ${ }^{1}$, N. Guañabens ${ }^{1}$, P. Peris ${ }^{1}$ ${ }^{1}$ Metabolic Bone Diseases Unit, Department of Rheumatology, ${ }^{2}$ Department of Nuclear Medicine; ${ }^{3}$ Department of Immunology, HOSPITAL CLÍNIC, Barcelona, Spain

Background: The trabecular Bone Score (TBS) is a novel gray-level textural analysis measurement that can be applied to DXA images to estimate trabecula microarchitecture and has been shown to be related to direct measures of bone microarchitecture and fracture risk. Osteogenesis impefecta $(\mathrm{OI})$ is a congenital bone disease characterised by a low bone mineral density (BMD) and poor bone quality and strength. The usefulness of TBS in OI has been scarcely evaluated.

Objectives: To analyse the clinical usefulness of TBS determination in patients with $\mathrm{OI}$ and its relation with anthropometric and clinical features (especially concerning skeletal fractures and BMD results).

Methods: Twenty-four patients (18 F:6 M) with Ol with a mean age of $38 \pm 15$ years $^{19-63}$ attending a Metabolic Bone Disease Unit were included. The clinical reports of the patients were reviewed, with especial attention to the clinical features (weight, height and body mass index [BMI]), previous fractures, disease severity, associated mutations and treatments received. Lumbar spine (LS), total hip $(\mathrm{TH})$, and femoral neck (FN) BMD were measured using DXA equipment (Lunar) in all patients. TBS was analysed in LS, and the results were classified in three categories ${ }^{1}$ : TBS $>1.310$ (normal), TBS 1.230-1.310 (partially degraded microarchitecture), TBS $<1.230$ (degraded microarchitecture). TBS values were compared with a healthy control group of similar age and gender.

Results: $5 / 24$ patients $(21 \%)$ had a degraded microarchitecture, $4(17 \%)$ a partially degraded microarchitecture and 15 (63\%) normal TBS. All patients with TBS $<1230$ were over 40 years old. $21 / 24$ patients had a previous history of multiple fractures. Regarding BMD, $54 \%$ of the patients had osteoporosis, $42 \%$ osteopenia and one had normal values. Most patients had a mutation in the COL1A gene $(63 \%)$. A correlation was observed between TBS and age $(r=-0.5, p=0.006)$ and LS BMD ( $r=0.5, p=0.014)$, showing a trend to significance with BMI $(r=-0.4$ $\mathrm{p}=0.058$ ). No significant differences were observed on comparing TBS in patients and controls (1.321 vs. $1.391, p=N$.S.).

Conclusions: TBS measurement does not seem to be useful for evaluating bone strength in patients with OI. Despite most patients presenting a history of multiple fractures, only $21 \%$ showed degraded microarchitecture with TBS.

\section{REFERENCE:}

[1] McCloskey EV, Odén A, Harvey NC, et al. J Bone Miner Res 2016;31:940-8.

Disclosure of Interest: None declared

DOI: 10.1136/annrheumdis-2018-eular.4883 